\title{
Telomerase activity in transthoracic fine needle biopsy aspirates as a marker of peripheral lung cancer
}

\author{
T Targowski, ${ }^{1}$ K Jahnz-Różyk, ${ }^{1}$ T Szkoda, ${ }^{2}$ S From, ${ }^{1}$ N Oandil, ${ }^{3}$ T Płusa ${ }^{1}$
}

${ }^{1}$ Department of Internal Medicine, Pneumonology and Allergology Military Institute of Health Service, Warsaw, Poland; ${ }^{2}$ Department of Virology, National Institute of Hygiene, Warsaw, Poland; ${ }^{3}$ Department of Pathomorphology Mazovian Centre of Lung Diseases and Tuberculosis, Otwock, Poland

Correspondence to:

Dr T Targowski, Military Institute of Health Service, Department of Internal Medicine

Pneumonology and Allergology, Szaserów Str. 126, 00-909

Warsaw, Poland; targowski. tomaszxl@wp.pl

Received 24 April 2007 Accepted 12 October 2007 Published Online First 26 November 2007

\begin{abstract}
Background: A study was undertaken to evaluate the usefulness of telomerase activity assay in transthoracic fine needle biopsy (TFNB) aspirates collected from peripheral tumours of the lung in predicting the malignant aetiology of lung infiltrations.

Methods: 100 patients with a peripheral infiltration of the lung underwent TFNB of the focal lesion. The aspirates were subjected to standard cytological evaluation. Telomerase activity in the specimens was determined with the PCR-ELISA PLUS method. The sensitivity, specificity, accuracy and predictive value of TFNB were calculated for cytological examination of aspirates alone and cytological examination with additional telomerase activity assessment.
\end{abstract}

Results: Lung cancer was newly diagnosed in 84 subjects and benign peripheral lesions were found in 16 . During the first TFNB, lung cancer was identified in 56 cases of cancer (66.7\%) while increased telomerase activity was found in 61 cancer aspirates (72.6\%). No subject with a benign infiltration had a false positive result from cytological examination, but in one case $(6.25 \%)$ increased telomerase activity was observed. The diagnostic sensitivity, accuracy and negative predictive value of the combination of cytological examination and telomerase activity assay in TFNB specimens were significantly higher than for cytological examination alone (89.3\% vs $66.7 \%, p=0.0004 ; 90 \%$ vs $72 \%, p=0.001$; $62.5 \%$ vs $36.4 \%, p=0.039$ ), but a combination of the two examinations was associated with a lower specificity of TFNB (96.9\% vs 100\%, $p=0.002$ ).

Conclusion: Detection of telomerase activity in aspirates taken during TFNB of a peripheral lung infiltration should be considered as an indication of the risk of malignancy in cases with false negative cytological results.

Suspicious lung infiltrations, including malignant tumours, are frequently located peripherally in the lung parenchyma and transthoracic fine needle biopsy (TFNB) appears to be a useful method of cytological diagnosis of such lesions. ${ }^{1-3}$ However, TFNB has drawbacks including the small amount of cells obtained during the procedure, a moderately high risk of false negative results and complications such as pneumothorax. ${ }^{4-7}$ The absence of neoplastic cells in smears collected during fine needle biopsy is not sufficient evidence to consider the lung lesions to be benign and cannot be used to abandon further diagnosis of the lung infiltration. Additional assessment of specific markers of malignant disorders in TFNB aspirates could improve the sensitivity of TFNB and diminish the risk that malignant lung cancer will remain unrecognised.
Telomerase, a ribonucleic enzyme responsible for uncontrolled proliferation of cancer cells, is a highly specific molecular marker of malignant diseases. $^{8}$ It is believed that increased telomerase activity could be a helpful indicator of the malignant aetiology of tumours and a prognostic factor for cancer development and patient survival. ${ }^{8}$ This study was undertaken to assess how evaluation of telomerase activity in aspirates from peripheral lung infiltrations influences the diagnostic value of TFNB.

\section{METHODS}

The study involved 100 patients with a peripheral (located beyond the field of vision of a bronchoscope) focal infiltration of the lung who were qualified for TFNB. All the patients underwent CT scanning of the lung. Indications for TFNB included (1) solid tumours with irregular contours, non-calcified or with peripheral, disseminated or spotted calcifications; (2) perimediastinal location of lesions (which could suggest small cell lung cancer); (3) enlargement of mediastinal lymph nodes (patients who were candidates for preoperative treatment in case of lung cancer); (4) distant extrapulmonary changes (in brain, bone or liver) which might appear to be metastatic foci of primary lung tumour.

TNFB was performed using a Becton Dickinson needle $0.7 \mathrm{~mm}$ in diameter under fluoroscopic control. After radiologically-guided placement of the needle in the tumour, an aspirate was taken and divided into two equal specimens for cytological and molecular examinations. The aspirates for the cytological examination were smeared on defatted slides fixed in 95\% ethyl alcohol and stained with eosin and haematoxylin. The specimens were evaluated by two pathologists separately and the final diagnosis was established by consensus between them. The pathologists were not informed about the level of telomerase activity in the specimens at any time before they reached the final diagnosis.

The aspirates for assessment of telomerase activity were immediately placed in $1 \mathrm{ml}$ probes and deeply frozen at $-70^{\circ} \mathrm{C}$. The PCR-ELISA ${ }^{\text {PLUS }}$ method (Roche Molecular Biochemicals, Mannheim, Germany) was used for the assessment of telomerase activity as described below. ${ }^{10}$

After defrosting, the aspirates were homogenised in $200 \mu \mathrm{l}$ ice-cooled Lysis reagent. The lysate was centrifuged at $16000 \mathrm{~g}$ for $20 \mathrm{~min}$ at $4^{\circ} \mathrm{C}$. $175 \mu \mathrm{l}$ of the supernatant was gently removed and its protein concentration measured with the Bio Rad 
Protein Assay Kit. Each supernatant was divided into two aliquots. One, which was inactivated at $85^{\circ} \mathrm{C}$ for $10 \mathrm{~min}$, was used as a negative control while the other one was used to evaluate telomerase activity. $3 \mu \mathrm{g}$ of protein extract was used for each assay. Assessment of telomerase activity was done according to the Telomeric Repeat Amplification protocol (TRAP) method which consists of amplification of telomeric sequences added by telomerase to the $3^{\prime}$ end of biotin-labelled synthetic primer. These elongation products, as well as the internal standard which constituted a positive control included in the same reaction vessel, were amplified using the appropriate primers. The PCR products were split into two aliquots, denaturated and hybridised to digoxigenin-labelled probes specific for the telomeric repeats and for the IS, respectively. The resulting products were immobilised via the biotin label to a streptavidin-coated microtitre plate. Immobilised amplicons were then detected with an anti-digoxigenin antibody conjugated to horseradish peroxidase and the sensitive peroxidase substrate. Absorbance of the samples was measured using an ELISA reader with a wavelength of $450 \mathrm{~nm}$. Samples were considered to be telomerase-positive if the difference in absorbance was higher than the background activity.

The sensitivity, specificity, accuracy and predictive value of TFNB in diagnosing malignant and benign aetiologies of lung infiltrations were assessed. These parameters were calculated for cytological examination alone and for cytological examination combined with assessment of telomerase activity of aspirates. Aspirates from malignant tumours with cancer cells and/or the presence of telomerase activity were considered as true positives while aspirates from benign infiltrations of the lung without cancer cells and/or telomerase activity were regarded as true negatives. Aspirates taken from malignant tumours without cancer cells and/or telomerase activity were considered as false negatives and aspirates from benign infiltrations with recognised cancer cells in the aspirates or increased telomerase activity were regarded as false positives.

Differences in the sensitivity, specificity, accuracy, positive and negative predictive values of TFNB were evaluated with $\chi^{2}$ and Fisher tests. The concordance between the presence or absence of telomerase activity and the presence or absence of cancer cells in aspirates was calculated. The Mann-Whitney test was used to compare the mean size of suspected infiltrations in patients in whom telomerase activity results agreed with cytological findings and patients in whom these results were not consistent. The confidence interval (CI) was set at $95 \%$.

\section{RESULTS}

The mean size of suspicious lesions was $2.4 \mathrm{~cm}$ (95\% CI 2.2 to 2.5). Lung cancer was confirmed in 84 of the 100 participants while in 16 the lesions were found to be benign.

After cytological examination of the aspirates collected during the first TFNB, cancer was identified in 56 patients $(66.7 \%)$, including $52(61.9 \%)$ cases of non-small cell lung cancer
(NSCLC) and $4(4.8 \%)$ cases of small cell lung cancer (SCLC). Moderate atypia was observed in 5 cases (5.9\%) and smears from 23 subjects $(27.4 \%)$ were non-diagnostic (ie, they contained necrotic, purulent or epithelial cells or cells with mild atypia or other non-specific blood cells). Of the 28 patients without a diagnosis of cancer after evaluation of the smears from the first TFNB, 10 (12\%) were found to have NSCLC after subsequent aspirations (performed within 7 days of the first TFNB with a $0.9 \mathrm{~mm}$ gauge needle), in 15 (17.9\%) a histological diagnosis of NSCLC was established after open lung biopsy and in $2(2.4 \%)$ after a subsequent procedure (1 resection of brain metastases, 1 bronchial brushing during bronchofibroscopy), and in 1 subject SCLC was diagnosed after surgical biopsy. Increased telomerase activity was observed in $61(72.6 \%)$ of 84 aspirates from peripheral malignant tumours of the lung, including 19 cases without cancer cells in the smears. In only 9 cases were neither cancer cells nor telomerase activity found in aspirates derived from peripheral malignant tumours of the lung (table 1).

Of the 16 subjects with benign focal infiltrations, 8 had nonspecific inflammatory infiltrations, 4 had tuberculomas, 3 had active tuberculosis and 1 had hamartoma. In 1 case of inflammation, atypia was observed. There were no false positive cytological results in this group of patients, but increased telomerase activity was observed in 1 case of non-specific inflammation of the lung (with atypical cells). The concordance between the presence or absence of telomerase activity and the presence or absence of cancer cells in aspirates was $66 \%$.

There were no significant differences in the mean size of suspected infiltrations between the patients in whom telomerase activity results agreed with cytological findings and those in whom these results were not in agreement $(-2.4 \mathrm{~cm}$ (95\% CI 2.3 to 2.6 ) and $2.3 \mathrm{~cm}$ (95\% CI 2.1 to 2.5$)$, respectively; $p=0.48)$.

It was found that the addition of telomerase activity assessment to cytological examination in TFNB aspirates from peripheral lung infiltrations significantly improved the sensitivity, accuracy and negative predictive value of fine needle biopsy, but it was associated with a decrease in the specificity of TFNB (table 2).

\section{DISCUSSION}

Owing to the easy accessibility of imaging techniques, peripheral focal lesions of the lung of unknown aetiology are often observed. ${ }^{11}$ In order to determine the malignant or benign character of these lesions, TFNB is performed. ${ }^{2}$ However, TFNB is an oligo-cell technique with limited sensitivity. ${ }^{413}$ In addition, it is an invasive method with the risk of serious complications such as pneumothorax which, under unfavourable conditions, occur in half of cases. ${ }^{5}$

Telomerase activity assessment in TFNB aspirates could be a helpful tool in the differential diagnosis of malignant and benign focal lesions of the lung. Although sporadic cases of high telomerase expression in a fatal course of pneumonia and cystic fibrosis have been described, ${ }^{14}$ a high level of telomerase activity

Table 1 Telomerase activity and cytological examination results in subjects with lung cancer

\begin{tabular}{llllll}
\hline \multirow{2}{*}{$\begin{array}{l}\text { Telomerase } \\
\text { activity }\end{array}$} & \multicolumn{5}{l}{ Results of cytological examination of TFNB aspirates } \\
\cline { 2 - 6 } & NSCLC & SCLC & Atypical cells & Non-diagnostic* & \multicolumn{1}{l}{ All } \\
\hline Negative & $14(26.9)$ & $0(0)$ & $1(25)$ & $8(34.8)$ & $23(27.4)$ \\
Positive & $38(73.1)$ & $4(100)$ & $4(75)$ & $15(65.2)$ & $61(72.6)$ \\
\hline
\end{tabular}

TFNB, transthoracic fine needle biopsy; NSCLC, non-small cell lung cancer; SCLC, small cell lung cancer.

Values are presented as $\mathrm{n}(\%)$.

${ }^{*}$ Mild atypia, necrotic, purulent, bronchial epithelial cells, granulocytes, erythrocytes, etc. 
Table 2 Diagnostic value of TFNB in lung cancer

\begin{tabular}{lllll}
\hline & $\begin{array}{l}\text { Cytological } \\
\text { examination alone (1) }\end{array}$ & $\begin{array}{l}\text { Telomerase activity } \\
\text { assessment alone (2) }\end{array}$ & $\begin{array}{l}\text { Cytological examination + } \\
\text { telomerase activity } \\
\text { assessment (3) }\end{array}$ & $\begin{array}{l}\text { p Value 1 vs 3 } \\
\text { ( } \chi^{2} \text { test) }\end{array}$ \\
\hline $\begin{array}{l}\text { Sensitivity (\%) } \\
\text { Specificity (\%) }\end{array}$ & $66.7(56.1$ to 75.8$)$ & $72.6(62.3$ to 81.0$)$ & $89.3(80.9$ to 94.3$)$ & 0.0004 \\
$\begin{array}{l}\text { Accuracy (\%) } \\
\begin{array}{l}\text { Negative predictive } \\
\text { value (\%) }\end{array}\end{array} \quad 36.0(62.5$ to 100$)$ & $93.8(71.7$ to 98.9$)$ & $96.9(84.3$ to 99.5$)$ & $0.002^{*}$ \\
$\begin{array}{l}\text { Positive predictive } \\
\text { value (\%) }\end{array}$ & $100(96.0(66.8$ to 83.3$)$ & $90(82.6$ to 94.5$)$ & 0.001 \\
\hline
\end{tabular}

Values are shown as mean (95\% confidence interval).

*Fisher test.

is characteristic of all cells of malignant tumours and is responsible for their uncontrolled proliferation. ${ }^{8}$ In healthy mature organisms, telomerase activity is detectable in rapidly dividing cells such as germ cells, epithelial cells, lymphocytes or activated fibroblasts $^{8}{ }^{15}$ but it is still significantly lower than in malignant neoplastic tissues. For example, it has been shown that the activity of telomerase in oesophageal cancer cells is 600 times higher than in diploid fibroblasts. ${ }^{16}$

In this study, increased telomerase activity was found in $72.6 \%$ of aspirates taken from malignant tumours of the lung, including 19 cases with no cancer cells visible in the smears. The assessment of telomerase activity, performed in addition to cytological examination of aspirates, significantly improved the sensitivity, accuracy and negative predictive value of TFNB, although it was associated with a small but statistically significant reduction in the specificity of TFNB owing to the presence of telomerase activity in one benign case. We cannot exclude the possibility that dividing the aspirates into two equal parts in order to perform the telomerase assay could slightly decrease the cytological sensitivity of TFNB, but the $66.7 \%$ sensitivity of the first TFNB achieved in this study is comparable with the results of other authors. ${ }^{412}{ }^{13}$ Some studies have achieved a sensitivity of more than $90 \%$ with TFNB, but these excellent results are reached only in tumour located directly by the thoracic wall and such biopsies are often associated with a high risk of pneumothorax or even false positive cytological results. ${ }^{5}$

Improvement in the negative predictive value from $36.4 \%$ to $62.5 \%$ means that the absence of cancer cells and lack of telomerase activity in the aspirate from a peripheral infiltration of the lung increases the probability that the pulmonary lesion is benign by almost twofold.

To date, only in breast tumours has a significant improvement in the sensitivity of TFNB been observed with the addition of telomerase activity assessment. ${ }^{17} 18$ A further advantage of the evaluation of telomerase activity in tumours is the possibility of identifying patients with a potentially unfavourable prognosis as the high level of telomerase activity is an unfavourable prognostic factor in lung carcinoma that is not related to histological type, cancer clinical stage, age, sex and smoking habit. ${ }^{9} 1920$

In summary, detection of telomerase activity in oligo-cell aspirates from peripheral tumours of the lung could be a helpful warning of a malignant origin of the lung infiltration when no cancer cells are found in the aspirates.

Funding: The study was financially supported by the Polish Ministry of Science. Competing interests: None.

Ethics approval: The study protocol was approved by the ethics committee of the Military Medical Chamber (No 59/2002).

\section{REFERENCES}

1. Westcott JL. Percutaneous transthoracic needle biopsy. Radiology 1988;169:593.

2. Lacasse Y, Plante J, Martel S, et al. Transthoracic needle biopsy in the diagnosis of solitary pulmonary nodules: a survey of Canadian physicians. J Thorac Cardiovasc Surg 2003;126:761-8.

3. Kandallu K, Grant TH, Stull MA, et al. Percutaneous needle biopsy of mediastinal and pulmonary masses with a CT-guided extrapleural approach. Radiology 1996;201:270.

4. Gudmundsdottir A, Reynisson K, Gudmundsson G. Computerized tomography guided percutaneous needle biopsies at Lanspitali University Hospital. Indications, complications and result. Laeknabladid 2005;91:917-21.

5. Ohno Y, Hatabu H, Takenaka D, et al. CT-guided transthoracic needle aspiration biopsy of small ( $\leqslant 20 \mathrm{~mm}$ ) solitary pulmonary nodules. AJR 2003;180:1665-9.

6. Olszewski W. Aspiration biopsy of intrathoracic lesions. Curr Diagn Patho/ 1995;2:146-52.

7. Wojtas G, Jahnz-Różyk K, Płusa T, et al. [Frequency of complications after transthoracic fine-needle biopsy of lung tumors]. Pol Merk Lek 2001;11:314-9.

8. Kim NW, Piatyszek MA, Prowse KR, et al. Specific association of human telomerase activity with immortal cells and cancer. Science 1994;266:2011-5.

9. Hiyama K, Hiyama E, Ishioka S, et al. Telomerase activity in small-cell and non-smallcell lung cancers. J Natl Cancer Inst 1995;87:895-902.

10. Anon. Telo TAGGG Telomerase PCR ELISAPLUS. Photometric enzyme immunoassay for quantitative determination of telomerase activity, utilizing the telomeric repeat amplification protocol (TRAP). Instruction manual. Mannheim, Germany: Roche Diagnostics, 1999.

11. Swensen JS, Jett JR, Hartman TE, et al. Lung cancer screening with CT: Mayo Clinic experience. Radiology 2003;226:756-61.

12. Larsheid RC, Thorpe PE, Scott WJ. Percutaneous transthoracic needle aspiration biopsy: a comprehensive review of its current role in the diagnosis and treatment of lung tumors. Chest 1998;114:704-9.

13. Sobolewski M, Włodarczyk R, Wojtas G, et al. [Fine needle biopsy in diagnosis of lung tumors]. Pol Merk Lek 1999;7:105-6.

14. Hiyama K, Ishioka S, Shay JW, et al. Telomerase activity as a novel marker of lung cancer and immune-associated lung diseases. Int J Mol Med 1998;1:545-9.

15. Harley CB, Futcher AB, Greider CW. Telomeres shorten during aging of human fibroblast. Nature 1990;345:458-60.

16. Shammas MA, Koley H, Beer DG, et al. Growth arrest, apoptosis, and telomere shortening of Barrett's-associated adenocarcinoma cells by a telomerase inhibitor. Gastroenterology 2004;126:1337-46.

17. Hiyama E, Saeki T, Hiyama K, et al. Telomerase activity as a marker of breast carcinoma in fine-needle aspirated samples. Cancer 2000;90:235-8.

18. Jin $\mathbf{S}$, Zhang $\mathbf{W}$, Teng $\mathbf{M}$, et al. Significance of telomerase activity detection by fineneedle aspiration in patients with breast cancer (abstract in English). Zhonghua Bing Li Xue Za Zhi 1999;28:334-6.

19. Strauss GM, Kwiatkowski DJ, Harpole DH, et al. Molecular and pathologic markers in stage I non-small cell carcinoma of the lung. J Clin Oncol 1995;43:1269-79.

20. Taga S, Osaki T, Oghami A, et al. Prognostic impact of telomerase activity in nonsmall cell lung cancers. Ann Surg 1999;230:715-20. 